\title{
Spin-polarized surface-emitting lasers
}

\author{
P. Bhattacharya*, M. Holub, and D. Saha \\ Solid-State Electronics Laboratory, Department of Electrical Engineering and Computer Science, \\ University of Michigan, Ann Arbor, MI 48109-2122, USA
}

Received 11 August 2006, accepted 25 August 2006

Published online 23 January 2007

PACS 42.55.Px, 72.25.Hg, 73.30.--y, 75.50.Bb, 81.15.Hi, 85.75.-d

We investigate the growth, fabrication, and characterization of an electrically-injected spin-polarized laser utilizing an $\mathrm{Fe} / n^{+}-\mathrm{Al}_{0.1} \mathrm{Ga}_{0.9} \mathrm{As}$ Schottky tunnel barrier for efficient spin injection into an InGaAs quantum well vertical-cavity surface-emitting laser. A threshold current reduction of $11 \%$ and maximum degree of circular polarization of $23 \%$ are observed for a $15 \mu \mathrm{m}$ mesa diameter laser at $50 \mathrm{~K}$. The lack of a threshold current reduction and circularly-polarized electroluminescence in similar nonmagnetic and Fe control lasers provides further evidence of spin injection.

() 2006 WILEY-VCH Verlag GmbH \& Co. KGaA, Weinheim

\section{Introduction}

Semiconductor spintronics - a new field of research exploring the interplay between magnetism and electronics - has received considerable attention at both experimental and theoretical levels with the ultimate goal of utilizing electron spin to make smaller, faster, and more versatile devices than those based solely on electron charge. The spin-polarized vertical-cavity surface-emitting laser (spin-VCSEL) promises a number of advantages over a conventional VCSEL. As a consequence of optical selection rules, spin-polarized electrons couple selectively to either the left- or right-circularly polarized lasing mode in a VCSEL such that pumping with a $100 \%$ spin-polarized current would result in a laser featuring circularly-polarized stimulated emission with half the threshold current. Additional advantages may include enhanced intensity and polarization stability, greater modulation bandwidth, polarization control, and reduced wavelenth chirp. The ability to controllably modulate the optical polarization of semiconductor lasers between orthogonal states in addition to its intensity would enable telecommunications networks with enhanced bandwidth. Other applications benefitting from polarization control in a VCSEL include optical computing and reconfigurable optical interconnects.

Optical orientation experiments using circularly-polarized light to orient electron spins have already demonstrated polarization control $[1,2]$ and threshold current reduction $[3,4]$ at both low and room temperatures; however, an electrically-pumped VCSEL is required for practical applications. Electrical spin injection has been demonstrated by a number of groups in spin-polarized light-emitting diodes (spin-LEDs), with ferromagnet/Schottky [5] and ferromagnet/insulating [6] tunnel barriers yielding the largest spin injection efficiencies thusfar. Here we report on the growth, fabrication, and characterization of an electrically-pumped spin-VCSEL utilizing an Fe/AlGaAs Schottky tunnel barrier for electron spin injection. A laser threshold current reduction of $11 \%$ with a maximal degree of circular polarization of $23 \%$ are demonstrated in a $15 \mu \mathrm{m}$ mesa diameter Fe spin-VCSEL at $50 \mathrm{~K}$.

\footnotetext{
" Corresponding author: e-mail: pkb@eecs.umich.edu, Phone: (734) 763-6678, Fax: (734) 763-9324
} 


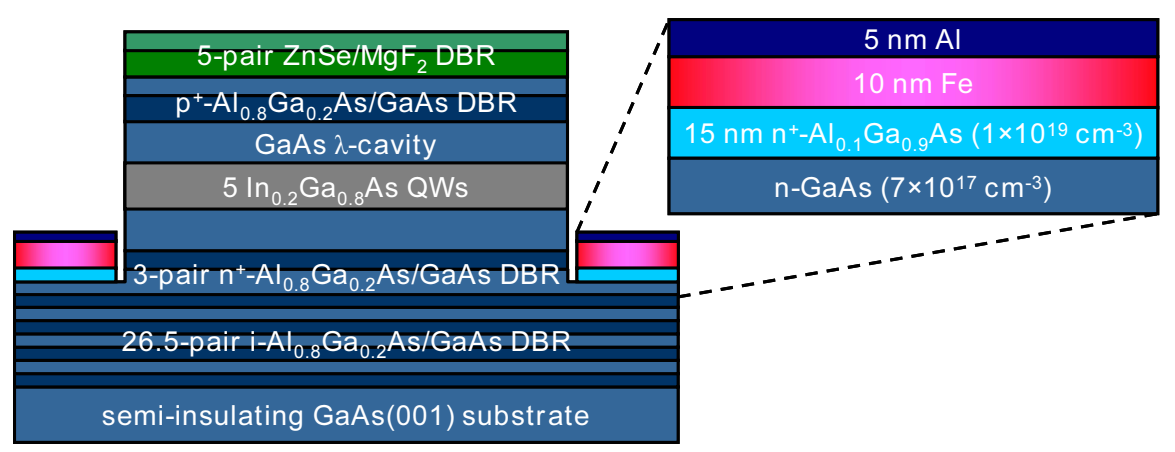

Fig. 1 Schematic cross section of the spin-laser heterostructure with a zoomed view of the regrown $\mathrm{Al} / \mathrm{Fe} / \mathrm{Al}_{0.1} \mathrm{Ga}_{0.9} \mathrm{As}$ Schottky tunnel barrier spin injector.

\section{Growth and fabrication}

The laser heterostructure, shown schematically in Fig. 1, was grown by molecular beam epitaxy on a semi-insulating GaAs(001) substrate. The spin-VCSEL consists of a 29.5 -pair, $n$-doped $\left(N_{\mathrm{D}}=7 \times 10^{17}\right.$ $\mathrm{cm}^{-3}$ ) $\mathrm{Al}_{0.8} \mathrm{Ga}_{0.2} \mathrm{As} / \mathrm{GaAs}$ distributed Bragg reflector (DBR) stack for the bottom mirror; a full-wave cavity spacer of GaAs with an active region comprised of five, $5 \mathrm{~nm} \operatorname{In}_{0.2} \mathrm{Ga}_{0.8}$ As quantum wells (QWs) separated by $10 \mathrm{~nm} \mathrm{GaAs}$ barriers; and a hybrid top mirror formed from one quarter-wave pair of $p$ doped $\left(N_{\mathrm{A}}=5 \times 10^{18} \mathrm{~cm}^{-3}\right) \mathrm{Al}_{0.8} \mathrm{Ga}_{0.2} \mathrm{As} / \mathrm{GaAs} \mathrm{DBR}$ and a 5-pair $\mathrm{ZnSe} / \mathrm{MgF}_{2}$ dielectric DBR stack. The peak reflectivities of the top and bottom mirrors are properly tailored for top-side emission. Since the electron spin relaxation time in InGaAs QWs is short (a few picoseconds) at room temperature [7], the spin-VCSEL was designed for low-temperature operation through introduction of a suitable gain offset. As shown in Fig. 2, a $16 \mathrm{~nm}$ red-shift of the gain peak wavelength relative to the laser cavity resonance wavelength at room temperature yields a minimum threshold current density of $806.2 \mathrm{~A} / \mathrm{cm}^{2}$ at $150 \mathrm{~K}$.

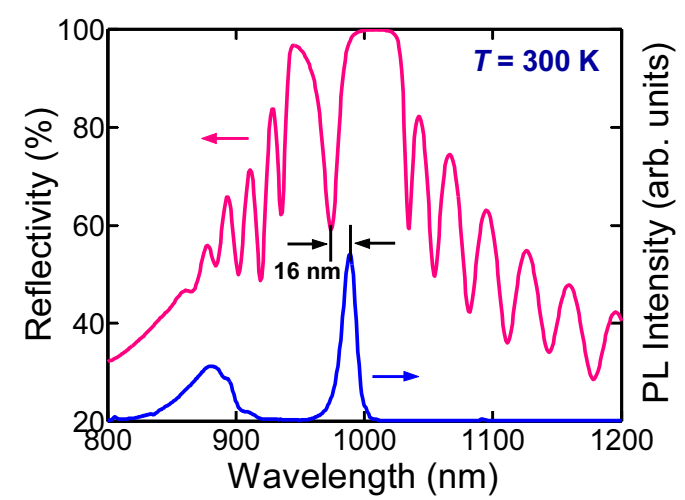

(a)

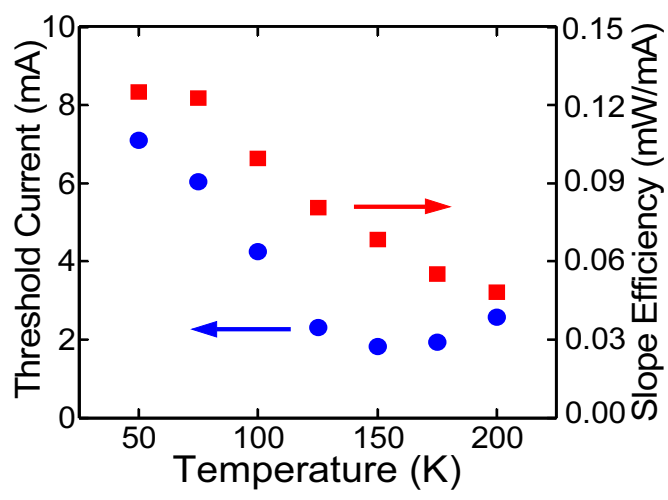

(b)

Fig. 2 (a) Reflectivity and photoluminescence (PL) spectra of the as-grown laser heterostructures showing a $16 \mathrm{~nm}$ gain offset at room temperature, and (b) temperature dependence of the threshold current and slope efficiency for a $20 \mu \mathrm{m}$ mesa diameter spin-polarized VCSEL.

Airpost spin-VCSELs were fabricated with mesa diameters varying from 15-30 $\mu \mathrm{m}$ using standard optical lithography, wet etching, polyimide planarization and passivation, metallization, and dielectric electron-beam evaporation techniques. An Fe $/ n^{+}-\mathrm{Al}_{0.1} \mathrm{Ga}_{0.9} \mathrm{As}$ Schottky barrier spin injector was deposited using an epitaxial re-growth step following mesa definition. An $\sim 2 \mu \mathrm{m}$ thick $\mathrm{SiO}_{2}$ layer deposited using plasma-enhanced chemical-vapour deposition was patterned to both reveal the $n$-GaAs contact 
layer and protect the mesa top surfaces and sidewalls during regrowth. After a high temperature surface preparation treatment to remove the native oxide formed on the exposed $n$-GaAs surface, a $15 \mathrm{~nm} n^{+}$$\mathrm{Al}_{0.1} \mathrm{Ga}_{0.9} \mathrm{As}\left(N_{\mathrm{D}}=1 \times 10^{19} \mathrm{~cm}^{-3}\right)$ layer was deposited at $610^{\circ} \mathrm{C}$. The substrate temperature was then ramped down and allowed to stabilize at a very low temperature $\left(\sim 40^{\circ} \mathrm{C}\right)$ in order to minimize potential intermixing at the Fe/AlGaAs interface, which can drastically reduce the spin injection efficiency due to spin-flip scattering from randomly oriented Fe ions [8]. An $\sim 10 \mathrm{~nm}$ Fe layer was deposted in an As-free environment using a growth rate of $0.35 \mathrm{~nm} / \mathrm{hr}$ and was subsequently capped with an $\sim 5 \mathrm{~nm}$ Al film to prevent oxidation of the Fe spin injector. Finally, the regrown material and protective $\mathrm{SiO}_{2}$ layer were removed from the mesas. Special care was taken to ensure that the regrown material was completely removed from the mesa sidewalls using wet etchants. Two control VCSELs were also fabricated to separate real from spurious contributions to the electroluminescence polarization, which is necessary for unabmiguosly verification of spin injection. The control devices are: (1) a nonmagnetic VCSELs with nonmagnetic $n$-ohmic metallization replacing the regrown $\mathrm{Fe} / \mathrm{Al}_{0.1} \mathrm{Ga}_{0.9} \mathrm{As}$ Schottky tunnel barriers and (2) Fe control VCSELs in which the regrown $10 \mathrm{~nm}$ Fe and $5 \mathrm{~nm} \mathrm{Al} \mathrm{layers} \mathrm{are} \mathrm{deposited} \mathrm{atop} \mathrm{nonmag-}$ netic $n$-ohmic metallization. All other design parameters remain identical to the Fe spin-VCSEL.

\section{Results and discussion}

The spin-VCSELs were mounted in a magneto-optical cryostat and characterized in the Farday geometry with a variable magnetic field applied along the surface normal. The electroluminescence spectrum exhibitied a full width at half maximum of $0.298 \mathrm{~nm}(0.4 \mathrm{meV})$ at $28 \mathrm{~K}$ as measured using a silicon charge coupled device attached to a spectrometer as shown in Fig. 3(a). Light versus current measurements were taken as a function of magnetic field using a silicon photodetector and current preamplifier for spinVCSELs under continuous-wave (CW) operation. Any change in laser threshold current would manifest as a change in total light intensity at a particular current bias point, and the laser threshold reduction may be inferred using the relationship $\Delta I_{t h}=\Delta L / \eta_{D}$, where $\mathrm{L}$ and $\eta_{D}$ are the total light intensity and differential slope efficiency, respecctively. As the magnetic field is increased from 0 to $5 \mathrm{kG}$, a threshold current reduction of $\Delta I_{t h} / I_{t h}=3.8 \%$ and, consequently, an emission intensity enhancement of $8.5 \mu \mathrm{W}$ are ob-

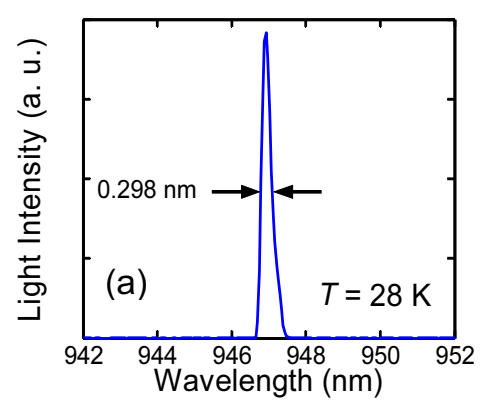

Fig. 3 (a) Electroluminescence spectrum of the Fe spin-polarized VCSEL taken at $28 \mathrm{~K}$ showing a full width at half maximum of 0.298 nm. (b) Percent threshold current reduction as a function of external magnetic field for spin-polarized and nonmagnetic VCSELs at $50 \mathrm{~K}$. Light versus current characteristics for a (c) spin-polarized and (d) nonmagnetic VCSEL taken at $50 \mathrm{~K}$. Inset shows the magnetic field dependence of the light intensity at a continuous-wave bias of $5 \mathrm{~mA}$.
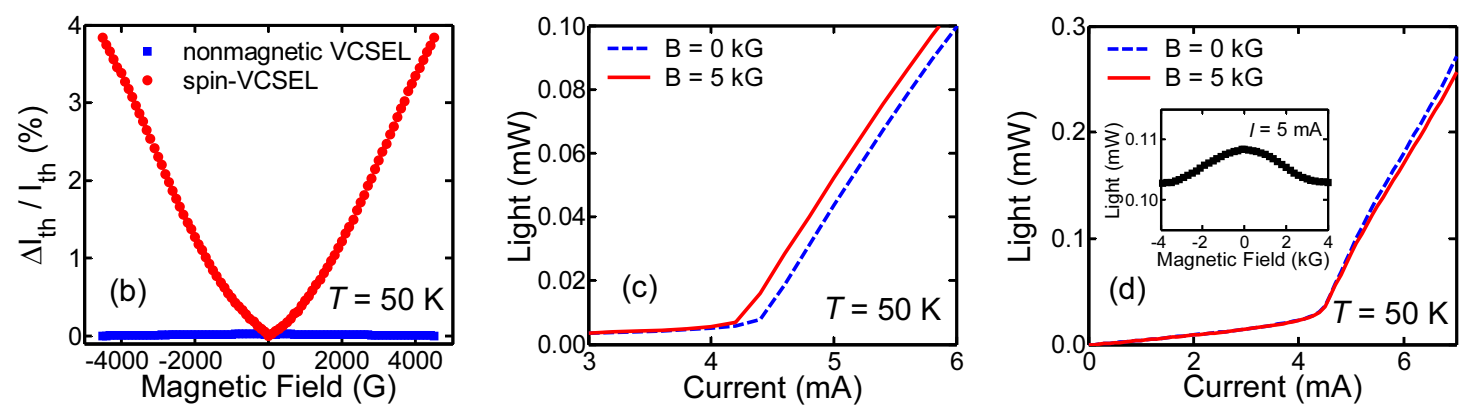
served for a $17 \mu \mathrm{m}$ mesa diameter spin-VCSEL biased at $1.2 \times I_{t h}$ [Figs. 3(b) and 3(c)]. A negligible change in threshold current and emission intensity near threshold were observed for the nonmagnetic control VCSEL [Fig. 3(d)] as well as for the Fe control VCSEL, which provides convincing evidence of electron spin injection in our laser heterostructure. When biased well above threshold, there is a general trend toward slightly lower optical powers for increasing magnetic field as shown in the inset to Fig. 3(d), an effect which is present in both nonmagnetic and spin-polarized VCSELs.

For further evidence of spin injection, the degree of circular polarization of the laser emission under $\mathrm{CW}$ bias was analyzed using a photoelastic modulator (PEM) operating at $50 \mathrm{kHz}$, a Glan-Thompson linear polarizer, and a silicon avalanche photodetector. The output of the photodetector is passed through a low-noise preamplifier and subsequently monitored by a digital lock-in amplifier referenced to the PEM modulation frequency. The Fe spin-VCSELs were mounted in a magneto-optical cryostat equipped with a $7 \mathrm{~T}$ superconducting magnet and biased $\mathrm{CW}$ near threshold $\left(1.2 \times I_{t h}\right)$. The degree of circular polarization, $\Pi_{\mathrm{C}}$, for a $15 \mu \mathrm{m}$ mesa diameter Fe spin-VCSEL is seen to very closely follow the out-of-plane magnetization of a $10 \mathrm{~nm}$ Fe film obtained by independent superconducting quantum interference device magnetometry measurements, reaching a saturation value of $\sim 23 \%$ at $50 \mathrm{~K}$ as shown in Fig. 4 . The sign of $\Pi_{\mathrm{C}}$ is consistent with majority spin injection as is typically observed for spin-LED containing $\mathrm{Fe} / \mathrm{AlGaAs}$ or $\mathrm{Fe} / \mathrm{Al}_{2} \mathrm{O}_{3}$ tunnel injectors [5, 9]. Since the Fe layer is incorporated outside of the resonant cavity, a parasitic polarization arising from magnetic circular dichroism (MCD) is absent in our device as longitudinal and transverse optical confinement are ensured by the DBR mirrors and airpost geometry, respectively, such that the emission never passes through the magnetic layer. Nevertheless, the parasitic contribution arising from MCD was investigated in our Fe spin-VCSEL via magneto-photoluminescence measurements using linearly-polarized laser excitation to generate unpolarized electro-hole pairs directly in the laser active region and assessing the degree of circular polarization of the resulting emission. A negligible degree of circular polarization was observed in this control experiment, which indicates that a parasitic contribution from MCD is absent in the Fe spin-VCSEL.

The threshold reduction as a function of magnetic field was determined from light intensity measurements for a $15 \mu \mathrm{m} \mathrm{Fe} \mathrm{spin-VCSEL} \mathrm{at} 50 \mathrm{~K}$. As shown in Fig. 4(b), the threshold current reduction increases with increasing magnetic field and saturates for magnetic field values above the out-of-plane saturation field for $\mathrm{Fe}(\sim 2.2 \mathrm{~T})$ reaching a maximal value of $\sim 11 \%$. The observed saturation of the threshold current reduction strongly indicates that the reduction is due to spin injection from the Fe/AlGaAs Schottky tunnel barrier contact.

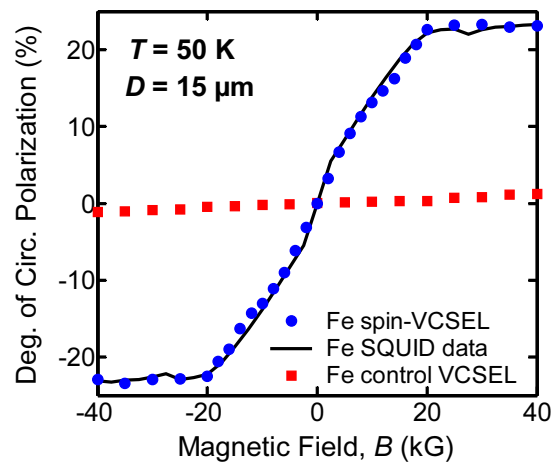

(a)

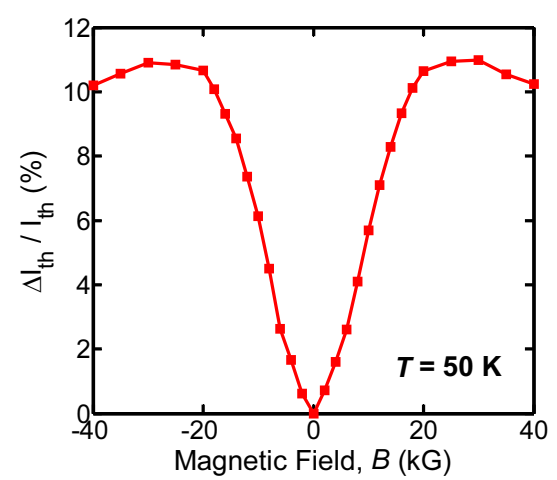

(b)

Fig. 4 (a) Degree of circular polarization versus external magnetic field measured at $50 \mathrm{~K}$ for a $15 \mu \mathrm{m}$ mesa diameter Fe spin-polarized VCSEL and Fe control VCSEL. The normalized out-of-plane magnetization curve for a $\sim 10$ $\mathrm{nm}$ Fe layer is shown for comparison. (b) Threshold reduction inferred from total light intensity measurements performed as a function of magnetic field for a $15 \mu \mathrm{m}$ mesa diameter Fe spin-polarized VCSEL at $50 \mathrm{~K}$. 
The optical selection rules for a strain quantum well suggest that $\Pi_{\mathrm{C}}=-\Pi_{\mathrm{S}}$ where $\Pi_{\mathrm{S}}$ is the active region spin polarization. This relationship has been widely employed to accurately quantify the spin polarization in spin-LEDs. A cursory application of this relationship to the present polarization data would suggest an active region spin polarization of $23 \%$. However, given the nonlinear dynamics in a laser, the simple relations derived from the optical selection rules to relate spin and optical polarizations in light-emitting diodes are likely invalid for the case of semiconductor spin-lasers. The spin anisotropy introduced in a spin-VCSEL either through optical orientation or electrical spin injection results in gain anisotropy for the two orthogonal circularly-polarized lasing modes. When biased near threshold, a small difference in spin polarization can result in a large difference between right- and left-circular polarization intensities if the gain coefficient for one mode is above the threshold gain required for lasing while the other is subcritical. Thus, spin information encoded onto the active region spin polarization can become amplified in a spin-VCSEL through a stimulated emission amplification process. The optical polarization anisotropy tends to decrease with increasing excitation when both modes are sufficiently pumped above threshold and will eventually disappear at the onset of gain saturation. Of course, the observance of gain and optical polarization anisotropy resulting from spin anisotropy requires that the spin relaxation time be greater than the carrier lifetime. Above threshold the carrier lifetime is on the order of a few tens of picoseconds and is limited primarily by the QW carrier capture time, which includes both diffusion in the barrier and capture by the well. Techniques such as QW intermixing [9] and impurity-mediated resonant tunnelling [10] have been shown experimentally to reduce the carrier capture time in InGaAs/GaAs QWs from $45 \mathrm{ps}$ to $20 \mathrm{ps}$ at $12 \mathrm{~K}$ and from $80-150 \mathrm{ps}$ to $600 \mathrm{fs}$ over the temperature range $10-150 \mathrm{~K}$, respectively. Application of such techniques to the design of spin-VCSELs could result in larger values of $\Pi_{\mathrm{C}}$ for a given, yet small, spin polarization. Alternatively, VCSELs utilizing GaAs QWs as the active medium could achieve a similar result since the measured spin relaxation time is approximately an order of magnitude greater in GaAs/AlGaAs QWs than for InGaAs/InP QWs [7].

\section{Conclusions}

We have investigated the properties of a spin-VCSEL where electron spin injection is achieved through a regrown $\mathrm{Fe} / \mathrm{Al}_{0.1} \mathrm{Ga}_{0.9} \mathrm{As}$ Schottky tunnel barrier. A maximum threshold current reduction of $11 \%$ is observed for a $15 \mu \mathrm{m}$ mesa diameter Fe spin-VCSEL along with a degree of circular polarization of $23 \%$ at $50 \mathrm{~K}$. The absence of a threshold current reduction and circular polarization in nonmagnetic and $\mathrm{Fe}$ control VCSELs strongly indicates that the observed effects in the Fe spin-VCSEL arise from spin injection, transport, and detection.

Acknowledgements This work is supported by the Office of Naval Research under Grant No. N00014-06-1-0025.

\section{References}

[1] S. Hallstein, J. D. Berger, M. Hilpert, H. C. Schneider, W. W. Rühle, F. Jahnke, S. W. Koch, H. M. Gibbs, G. Khitrova, and M. Oestreich, Phys. Rev. B 56, R7076 (1997).

[2] H. Ando, T. Sogawa, and H. Gotoh, Appl. Phys. Lett. 73, 566 (1998).

[3] J. Rudolph, D. Hägele, H. M. Gibbs, G. Khitrova, and M. Oestreich, Appl. Phys. Lett. 82, 4516 (2003).

[4] J. Rudolph, S. Dohrmann, D. Hägele, M. Oestreich, and W. Stolz, Appl. Phys. Lett. 112, 1649 (2005).

[5] A. T. Hanbicki, B. T. Jonker, G. Itskos, G. Kioseoglou, and A. Petrou, Appl. Phys. Lett. 80, 1240 (2002).

[6] X. Jiang, R. Wang, R. M. Shelby, R. M. Macfarlane, S. R. Bank, J. S. Harris, and S. S. P. Parkin, Phys. Rev. Lett. 94, 056601 (2005)..

[7] A. Tackeuchi, O. Wada, and Y. Nishikawa, Appl. Phys. Lett. 70, 1131 (1997).

[8] O. M. J. van’t Erve, G. Kioseoglou, A. T. Hanbicki, C. H. Li, B. T. Jonker, R. Mallory, M. Yasar, and A. Petrou, Appl. Phys. Lett. 84, 4334 (2004).

[9] L. V. Dao, M. B. Johnston, M. Gal, L. Fu, H. H. Tan, and C. Jagadish, Appl. Phys. Lett. 73, 3408 (1998).

[10] L. V. Dao, M. Gal, H. Tan, and C. Jagadish, Appl. Phys. Lett. 72, 2008 (1998). 